REFERENCES

[1] K. Krüger, et al. BMJ Open 2018

[2] K. Krüger, et al. Rheumatology International 39 (1) 2019

Disclosure of Interests: Klaus Krueger: None declared, Gerd Rüdiger Burmester Consultant for: Roche, Sanofi-Genzyme, Speakers bureau: Roche, Sanofi-Genzyme, Siegfried Wassenberg Grant/research support from: Roche Pharma, Consultant for: Abbvie, BMS, Chugai, Roche, Novartis, Pfizer, MSD, Speakers bureau: Abbvie, BMS, Pfizer, Janssen, Chugai, Celgene, Roche, Novartis, MSD, Astrid Thiele Consultant for: Biogen, Celgene, Chugai, Hexal, Janssen, Lilly, MSD, Novartis, Pfizer, UCB, Matthias Thomas Employee of: MSD Sharp \& Dohme GmbH Germany DOI: 10.1136/annrheumdis-2019-eular.3245

\section{SAT0167 INCREASED HIGH MOLECULAR WEIGHT ADIPONECTIN AND LEAN MASS DURING TOCILIZUMAB TREATMENT IN PATIENTS WITH RHEUMATOID ARTHRITIS. A 12 MONTHMULTICENTER STUDY}

Eric Toussirot ${ }^{1}$, Hubert Marotte ${ }^{2}$, Denis Mulleman ${ }^{3}$, Gregoire Cormier ${ }^{4}$, Fabienne Coury-Lucas ${ }^{5}$, Philippe Gaudin ${ }^{6}$, Emmanuelle Dernis ${ }^{7}$, Christine Bonnet ${ }^{8}$, Richard Damade ${ }^{9}$, Jean Luc Grauer ${ }^{10}$, Tassadit AitAbdesselam ${ }^{11}$, Caroline Guillibert Karras ${ }^{12}$, Frederic Lioté ${ }^{13}$, Pascal Hilliquin ${ }^{14}$ Antoinette Sacchi ${ }^{15}$, Jean Marie Berthelot ${ }^{16}$, Marc Puyraveau ${ }^{17}$, Gilles Dumoulin ${ }^{18}$ ${ }^{1}$ University Hospital, Rheumatology, BESANCON, France; ${ }^{2}$ University Hospital, Rheumatology, St Etienne, France; ${ }^{3}$ University Hopsital, Rheumatology, Tours, France: ${ }^{4} \mathrm{CH}$, Rheumatology, la Roche sur Yon, France; ${ }^{5}$ University Hospital, Rheumatology, Lyon, France; ${ }^{6}$ University Hospital, Rheumatology, Grenoble, France; ${ }^{7} \mathrm{CH}$, Rheumatology, Le Mans, France; ${ }^{8}$ University Hospital, Rheumatology, Limoges, France; ${ }^{9} \mathrm{CH}$, Rheumatology, Chartres, France; ${ }^{10} \mathrm{CH}$, Rheumatology, Montelimar, France; ${ }^{11} \mathrm{CH}$, Rheumatology, Meaux, France; ${ }^{12} \mathrm{CHSt}$ Joseph, Rheumatology, Marseille, France; ${ }^{13}$ University Hospital Lariboisiere, Rheumatology, Paris, France; ${ }^{14} \mathrm{CH}$ Sud Francilien, Rheumatology, Corbeil, France, ${ }^{15} \mathrm{CH}$, Rheumatology, Mantes le Jolie, France, ${ }^{16}$ University Hospital, Rheumatology, Nantes, France; ${ }^{17}$ University Hospital, biostatistics, BESANCON, France; ${ }^{18}$ University Hospital, Endocrine and metabolic chemistry, BESANCON, France

Background: Patients with rheumatoid arthritis (RA) have an increased risk of cardiovascular (CV) diseases. TNFa blockade in RA patients has been associated with weight gain, increase in fat mass and variations in serum adipokines. Adiponectin (Adp), a protein produced by adipocytes, plays a beneficial role in insulin sensitivity and CV disease prevention, especially its high molecular weight (HMW) isoform.

Objectives: To analyse the changes in serum adipokines and especially Adp (total and HMW) and body composition during TCZ therapy in RA Methods: Multicenter open-label study. All patients enrolled had active RA (2010 ACR/EULAR criteria and DAS28 $\geq 3$ 3.2) with previous inadequate response to a cSDMARDs and/or bDMARDs. They all were TCZ naïve and received TCZ IV. Patients were evaluated at baseline,1,3,6 and 12 months. Clinical assessment included body mass index (BMI), waist circumference, DAS28 and HAQ scores. Laboratory parameters of inflammation (ESR, CRP, IL-6), lipid parameters (total chol., LDL and HDL chol., triglycerides), metabolic parameters (glycemia, insulin), serum Adp (total and HMW), leptin, resistin, and ghrelin were measured at each time point. Body composition (lean mass, fat mass, $\%$ of fat, fat in the android and gynoid regions) was evaluated at baseline, 6, and 12 months (DEXA, Lunar GE). Our primary criteria was the changes in Adp (total and HMW) at 6 months.

Results: 107 patients $(78 \mathrm{~F}$; mean age \pm SD: $56.6 \mathrm{yr} \pm 13.5$; disease duration: $9.9 \mathrm{yr} \pm 8.1$; previous bDMARD: $64.5 \%$; corticosteroids: $69 \%$; concomitant CsDMARD: $72.8 \%$ ) were included. 97 were still on treatment at month 6 and 77 completed the study. Most of the patients (95\%) received TCZ $8 \mathrm{mg} / \mathrm{kg}$ by infusion. Patients were responding to TCZ with a significant DAS28 decrease between baseline and months 6 and 12 $(p<0.0001) . H A Q, E S R$, and CRP levels significantly decreased along the study $(\mathrm{p}<0.0001)$. BMI and abdominal circumference significantly increased at month 6 and 12, as well as lean mass $(p=0.0097$ at month 6 and 0.021 at month 12). Fat mas, $\%$ of fat and android fat did not change over the study. Total chol and LDL chol increased while glycaemia and insulin remained stable. Both total and HMW Adp increased from baseline to month 6 and month 12 (total Adp: baseline vs month 6: $p=0.055$; HMW Adp: baseline vs month $6: p=0.02$, baseline vs month 12 : $\mathrm{p}=0.057$ ). Serum leptin, resistin and ghrelin did not change during the follow-up.
Conclusion: These variations in Adp during TCZ treatment may have a positive impact on the $\mathrm{CV}$ risk of RA patients and may contribute to the protective role of TCZ against the CV burden in RA.

Acknowledgement: ADIPRAT study group This work was supported by a grant from ROCHE CHUGAI

Disclosure of Interests: ERIC TOUSSIROT Grant/research support from: Roche Chugai, Hubert MAROTTE: None declared, Denis Mulleman Speakers bureau: Pfizer, Novartis, Grifols, Gregoire CORMIER: None declared, Fabienne COURY-LUCAS: None declared, Philippe Gaudin Speakers bureau: Roche, Chugai, BMS, Abbvie, Servier, Pfizer, MSD UCB, ESAOTE, Genévrier, Janssen, Novartis, Lilly, Biogen, Amge, Emmanuelle Dernis: None declared, Christine BONNET: None declared, Richard DAMADE: None declared, Jean Luc GRAUER: None declared, Tassadit AIT-ABDESSELAM: None declared, Caroline GUILLIBERT KARRAS None declared, Frederic Lioté Grant/research support from: institutional grants from Grunenthal, Ipsen Pharma/Menarini, Novartis, SOBI for the European Crystal Network Workshops, Consultant for: Grunenthal, Novartis, Pascal Hilliquin Consultant for: Roche SAS, Antoinette SACCHI: None declared, Jean Marie BERTHELOT: None declared, Marc PUYRAVEAU: None declared, Gilles DUMOULIN: None declared DOI: 10.1136/annrheumdis-2019-eular.4675

\section{SAT0168 IMPACT OF AGE ON THE APPEARANCE OF ADVERSE EVENTSAT THE BEGINNING OF BIOLOGICAL TREATMENT: DATA FROM THE BIOBADASER 3.0 REGISTRY}

Paloma Vela-Casasempere ${ }^{1,2,3}$, Carlos Sánchez-Piedra ${ }^{4}$, Carolina Perez-Garcia ${ }^{5}$, Sara Manrique Arija ${ }^{6}$, María del Carmen Castro Villegas ${ }^{7}$, Blanca GarciaMagallon $^{8}$, Javier Manero ${ }^{9}$, Silvia Gomez-Sabater ${ }^{2}$, Rocio Caño-Alameda ${ }^{2}$, Fernando Sánchez-Alonso ${ }^{4}$,le Federico Diaz-Gonzaz ${ }^{4}$, Jesus GomeJuan zReino ${ }^{10} .{ }^{1}$ Alicante University General Hospital, Rheumatology, Alicante, Spain ${ }^{2}$ Isabial, Alicante, Spain; ${ }^{3}$ Miguel Hernandez University, Clinical Medicine, San Juan, Spain; ${ }^{4}$ Spanish Rheumatology Society, Research Unit, Madrid, Spain; ${ }^{5}$ Hospital del mar, Rheumatology, Barcelona, Spain; ${ }^{6}$ Hospital Carlos Haya, Rheumatology, Malaga, Spain; ' Hospital Reina Sofia, Rheumatology, Cordoba Spain; ${ }^{8}$ Hospital San Jorge, Rheumatology, Huesca, Spain; ${ }^{9}$ Hospital Miguel Servet, Rheumatology, Zaragoza, Spain; ${ }^{10}$ Hospital de Santiago, Rheumatology, Santiago de Compostela, Spain

Background: Currents medical advances are allowing patients with chronic arthritis to live to advanced ages. Although the risks of biological therapies in elderly patients have been previously evaluated, data are scarce since they are mainly derived from clinical trials, in which elderly populations are often underrepresented or event excluded (patients up to 75 years old). In addition, comparisons between such studies are difficult in the absence of a consensus in defining the groups' age.

Objectives: To evaluate the impact of age on the appearance of adverse events $(A E)$ in patients with rheumatic diseases (rheumatoid arthritis -RAankylosing spondylitis -AS- and psoriatic arthritis - PsA) at the start of biological treatment.

Methods: Multicenter prospective study in a real-world setting. Information was obtained from BIOBADASER, a national safety registry of patients with rheumatic diseases treated with biologics or targeted synthetic disease modifying anti-rheumatic drugs. For this analysis, all patients included in this registry since 2000 and diagnosed with RA, AS or PsA were included, and classified into four categories according to age at initiation of biologic treatment: young $(<25$ years-old), adults $(25-64$ yearsold), elderly (65-75 years-old) and very elderly (> 75 years-old). Data collected included: 1) patient's data 2) data on treatment and 3) data on AE. Proportions, means and standard deviations were used to describe the population. Poisson regression model was carried out to explore factors associated with the appearance of AEs. Crude and adjusted incidence rate ratios (IRRs) were calculated.

Results: A total of 2531 patients were included: 1154 RA (45.59\%), 680 PsA (26.87\%), and 697 AS (27.54\%). Age groups: there were 64 young patients $(2.52 \%), 2166$ adults $(85.57 \%), 243$ elderly adults (9.6\%), and 58 very elderly adults $(2.29 \%)$. Comorbidities increased with age, while smok ing rates decreased. Methotrexate use was similar in all age groups (53.99\% in the total sample), but corticosteroid treatment increased with age (young $27.7 \%$, adults $47 \%$, elderly adults $64.36 \%$ and the very elderly $70.21 \%$ ). $77.87 \%$ received anti-TNF treatment, and $22.13 \%$ other biological drugs. Poisson regression model showed an increased probability of suffering a first adverse event with increasing age regardless of the disease (IRR for AS: 1.04 (0.87-1.26); IRR for PsA: 1.08 (0.92-1.28)). 